\title{
Comparative assessment of image quality for coronary CT angiography with iobitridol and two contrast agents with higher iodine concentrations: iopromide and iomeprol. A multicentre randomized double-blind trial
}

\author{
Stephan Achenbach ${ }^{1}$. Jean-François Paul ${ }^{2}$ - François Laurent ${ }^{3,4}$. \\ Hans-Christoph Becker ${ }^{5} \cdot$ Marco Rengo $^{6} \cdot$ Jerome Caudron $^{7} \cdot$ Sebastian Leschka $^{8}$. \\ Olivier Vignaux ${ }^{9} \cdot$ Gesine Knobloch $^{10}$ • Giorgio Benea ${ }^{11}$ • Thomas Schlosser $^{12}$ • \\ Jordi Andreu ${ }^{13} \cdot$ Beatriz Cabeza $^{14}$ • Alexis Jacquier ${ }^{15}$. Miguel Souto $^{16} \cdot$ Didier Revel $^{17}$.

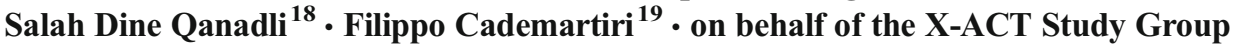

Received: 7 August 2015 /Revised: 19 May 2016 / Accepted: 23 May 2016/Published online: 7 June 2016

(C) The Author(s) 2016. This article is published with open access at Springerlink.com

\begin{abstract}
Objectives To demonstrate non-inferiority of iobitridol 350 for coronary CT angiography (CTA) compared to higher iodine content contrast media regarding rate of patients evaluable for the presence of coronary artery stenoses.

Methods In this multicentre trial, 452 patients were randomized to receive iobitridol 350 , iopromide 370 or iomeprol 400 and underwent coronary CTA using CT systems with 64detector rows or more. Two core lab readers assessed 18 coronary segments per patient regarding image quality (score $0=$ non diagnostic to $4=$ excellent quality), vascular
\end{abstract}

attenuation, signal and contrast to noise ratio (SNR, CNR). Patients were considered evaluable if no segment had a score of 0 .

Results Per-patient, the rate of fully evaluable CT scans was $92.1,95.4$ and $94.6 \%$ for iobitridol, iopromide and iomeprol, respectively. Non-inferiority of iobitridol over the best comparator was demonstrated with a $95 \% \mathrm{CI}$ of the difference of [-8.8 to 2.1], with a pre-specified non-inferiority margin of $-10 \%$. Although average attenuation increased with higher iodine concentrations, average SNR and CNR did not differ between groups.
Stephan Achenbach

stephan.achenbach@uk-erlangen.de

on behalf of the X-ACT Study Group

1 Department of Cardiology, Friedrich-Alexander-Universität Erlangen-Nürnberg, Ulmenweg 18, 91054 Erlangen, Germany

2 Department of Radiology, Centre Chirurgical Marie Lannelongue, Le Plessis Robinson, France

3 University of Bordeaux, Centre de Recherche Cardio-Thoracique de Bordeaux, U1045, F-33000 Bordeaux, France

4 CHU de Bordeaux, Service d'Imagerie Thoracique et Cardiovasculaire, F-33600 Pessac, France

5 Department of Clinical Radiology, University Hospital Grosshadern, Munich, Germany

6 Department of Radiological Sciences, Oncology and Pathology, Sapienza - University of Rome, ICOT Hospital, Latina, Italy

7 Department of Radiology, University Hospital of Rouen, Rouen, France

\footnotetext{
Department of Radiology, Saint Gallen Hospital, Saint Gallen, Switzerland

9 Department of Radiology, Cochin Hospital, Paris, France

10 Department of Radiology, La Charité, Berlin, Germany

11 Ospedale del Delta, Ferrara, Italy

12 Elisabeth-Krankenhaus Hospital, Essen, Gerrmany

13 Hospital Vall d'Hebron, Barcelona, Spain

14 Hospital Clinico San Carlos, Madrid, Spain

15 Department of Radiology, La Timone Adult Hospital, Marseille, France

16 Complejo Hospitalario Universitario, Santiago de Compostela, Spain

17 Department of Radiology, Louis Pradel Hospital, Lyon, France

18 Department of Radiology, University of Lausanne, Lausanne, Switzerland

19 Department of Radiology, Giovanni XXIII Hospital, Monastier di Treviso, Italy
} 
Conclusions With current CT technology, iobitridol $350 \mathrm{mg}$ iodine $/ \mathrm{ml}$ is not inferior to contrast media with higher iodine concentrations in terms of image quality for coronary stenosis assessment.

Key Points

- Iodine concentration is an important parameter for image quality in coronary CTA.

- Contrast enhancement must be balanced against the amount of iodine injected.

- Iobitridol 350 is non-inferior compared to CM with higher iodine concentrations.

- Higher attenuation with higher iodine concentrations, but no SNR or CNR differences.

Keywords Coronary computed tomography angiography . Image quality $\cdot$ Contrast media $\cdot$ Iodine concentration $\cdot$ Safety

\section{Introduction}

Coronary computed tomography angiography (coronary CTA) has become widely accepted in clinical practice [1-3]. Technology progress has increased the robustness and diagnostic performance of coronary CTA, resulting in improved image quality and lower radiation exposure [4-9].

The protocols for administration of intravenous contrast media (CM) are of major importance in coronary CTA, usually performed to identify coronary artery stenoses but also calcified and non-calcified plaques [10-13]. The optimal intravascular attenuation for coronary CT angiography is under debate [14, 15]. Several publications suggested that adequate opacification of the vessel lumen for the simultaneous identification of both calcified and non-calcified plaques requires a careful contrast injection protocol that achieves a lumen opacification of at least $300 \mathrm{HU}$ [15-17]. In principle, the Iodine Delivery Rate (IDR) should be the reference when using different compounds for intraluminal enhancement [18]. However, most centres do not use this approach in clinical practice.

Higher iodine concentration of the injected $\mathrm{CM}$ is associated with higher attenuation $[16,19]$. However, increasing the total amount of iodine injected could raise safety issues for patients at risk such as contrast induced nephropathy [20-22]. Therefore, adequate contrast enhancement must be balanced against the amount of iodine injected. Several studies have addressed the level of attenuation with different $\mathrm{CM}[14,23,24]$, but few studies evaluated the impact of their concentration on image quality [25]. Furthermore, whether differences less than $50 \mathrm{mg} / \mathrm{ml}$ of iodine concentration could affect image quality remains to be clarified. The present study compared a $\mathrm{CM}$ with iodine concentration of $350 \mathrm{mg}$ iodine/ml (iobitridol) to two $\mathrm{CM}$ with higher iodine concentrations (iopromide $370 \mathrm{mg} / \mathrm{ml}$ and iomeprol $400 \mathrm{mg} / \mathrm{ml}$ ) for coronary CTA. The main objective of the study was to demonstrate the statistical non- inferiority of iobitridol 350 compared to the best of the two comparators in terms of image quality and interpretability as measured by the rate of patients with CT scans evaluable for the identification of coronary artery stenosis.

\section{Material and methods}

\section{Study design and patient enrolment conditions}

This study was a non-inferiority, multicentre, randomized, double-blind, clinical trial on three parallel groups. Patients were included in 23 centres from five countries between November 2010 and September 2012 and randomized on a 1:1:1 ratio to undergo clinically indicated coronary CTA after injection of iobitridol, iopromide or iomeprol. The study was approved by each local ethics committees and the National Health Authorities. Written informed consent was obtained from each participating patient.

Symptomatic adult patients with suspected coronary artery disease (CAD), and scheduled for coronary CT angiography were enrolled in this study. Patients could not be included if they had both a contraindication to $\beta$-blocker medications and a baseline heart rate above 65 beats per minute (bpm). Additional reasons for exclusion were the presence of arrhythmias or nonsinus rhythm, coronary artery bypass grafts or stents, artificial heart valves, moderate to severe aortic valve stenosis, hyperthyroidism, clinical instability, severe renal failure or previous injection of any $\mathrm{CM}$ within 48 hours prior to the study.

\section{Patient preparation}

$\beta$-blockers were mandatory if heart rate was $>65 \mathrm{bpm}$. The specific drug, dose and mode of administration were selected according to site routine practice. A minimum dose of $0.8 \mathrm{mg}$ of sublingual nitroglycerine spray was mandatory immediately before the CT examination. Other pre-medication was permitted, given according to operator preference, and recorded.

\section{Injection of contrast media}

For each patient, one of three $\mathrm{CM}$ was delivered intravenously: Iobitridol $350 \mathrm{mg}$ iodine/ml (Xenetix ${ }^{\circledR}$, Guerbet, Aulnaysous-Bois, France), iopromide $370 \mathrm{mg} / \mathrm{ml}$ (Ultravist $\AA$, Bayer Healthcare, Berlin, Germany) and iomeprol $400 \mathrm{mg} / \mathrm{ml}$ (Iomeron ${ }^{\circledR}$, Bracco, Milan, Italy). Delivered volume and delivery rate of $\mathrm{CM}$ was consistent for the three $\mathrm{CM}$ but varied according to patient body weight (BW): $60 \mathrm{ml}$ injected at $4 \mathrm{ml} / \mathrm{s}$ for a BW $<60 \mathrm{~kg}, 75 \mathrm{ml}$ at $5 \mathrm{ml} / \mathrm{s}$ for a BW between 60 and $80 \mathrm{~kg}, 90 \mathrm{ml}$ at $6 \mathrm{ml} / \mathrm{s}$ for a BW $>80 \mathrm{~kg}$. Therefore, the iodine-delivery rate was lowest for iobitridol. $\mathrm{CM}$ was warmed and injections were followed by a $100 \%$ saline flush of $75 \mathrm{ml}$ administered at the same rate as the $\mathrm{CM}$. 


\section{Scan protocol}

All coronary CTAs were performed on systems with at least 64 detector rows (single or dual source). The tube voltage was adapted to the patient's BW depending on the equipment used in the site: either $120 \mathrm{kVp}$ for all patients or $120 \mathrm{kVp}$ for BW $\geq 80 \mathrm{~kg}$ and $100 \mathrm{kVp}$ for $\mathrm{BW}<80 \mathrm{~kg}$. Each patient first received a non-contrast acquisition for the quantification of coronary calcium, followed by a high-resolution contrast-enhanced acquisition. Non-contrast scans were acquired according to local protocol, mandating the use of $120 \mathrm{kV}$ tube voltage and $3 \mathrm{~mm}$ thickness of the reconstructed cross-sectional images.

Test bolus or bolus tracking [26, 27] as well as dose reduction techniques (e.g. mAs modulation, scan duration, tube current and pitch) were chosen according to site specific routine. Retrospective ECG-gating was always allowed, whereas prospectively ECG triggered data acquisition was only permitted for patients with $\mathrm{HR}<65 \mathrm{bpm}$.

Dose length product (DLP) and volume CT dose index (CTDIvol) were recorded for the non-enhanced and for the contrast-enhanced coronary CTA acquisition.

\section{Calcium scoring}

The calcium score [13] was calculated on-site. Patients were then classified using the following standard scale [28]: $1=$ normal (no calcium); 2 = mildly elevated (Agatston Score 1 to 100); $3=$ moderately elevated (Agatston Score 101 to 400); $4=$ highly elevated (Agatston Score 401 to 1000); 5 = severely elevated (Agatston Score >1000).

\section{Image reconstruction for coronary CTA}

Cross-sectional image data sets were reconstructed using the thinnest possible slice thickness and standard reconstruction kernels (i.e. medium-smooth) without implementation of iterative reconstruction algorithms in order to reduce softwarerelated attenuation variability. The choice of the best temporal window was left to the local operators. Original axial DICOM data were transferred for off-site reading purposes to a dedicated image core laboratory.

\section{Image evaluation for detection of stenosis}

All CTA images were assessed by two independent, experienced readers with more than 10 years of experience in coronary CT angiography, both fully blinded to the patients' clinical characteristics and the CM used.

The primary endpoint was the rate of patients with evaluable CT scans, i.e. CT scans that made it possible to identify or rule out coronary artery stenoses in all segments of the coronary tree with a reference diameter of $1.5 \mathrm{~mm}$ or more. Using a 5-point scale, off-site readers graded the quality of all 18 segments of the
Society of Cardiovascular Computed Tomography (SCCT) coronary segmentation model [29] (4 = excellent quality, full confidence without any doubts concerning the presence/absence of luminal stenosis; 3 = good quality, confidence concerning the presence/absence of luminal stenosis; 2 = moderate quality, relative confidence, with minor doubts concerning the presence/ absence of luminal stenosis; 1 = poor quality, some doubts concerning the presence/absence of stenosis; $0=$ non diagnostic, with relevant doubts concerning the presence/absence of stenosis). A patient's CT scan was considered as evaluable for identification of coronary artery stenosis if none of the 18 coronary segments had a score of 0 (except for segments graded 0 due to an occlusion located proximally, which did not make the patient non-evaluable).

As a secondary endpoint, a per-patient image quality score was computed off-site by averaging segment quality scores within patients.

\section{Attenuation, signal and noise measurements}

Arterial attenuation, signal-to-noise ratio (SNR) and contrastto-noise ratio (CNR) constituted secondary endpoints. Arterial vascular attenuation was measured off-site as follows: one region of interest (ROI) of $2 \mathrm{~mm}^{2}$ minimum size and located in the lumen of the LAD, the LCX, the RCA and the LM coronary artery; one ROI of $100 \mathrm{~mm}^{2}$ minimum size in the ascending aorta and one ROI of $50 \mathrm{~mm}^{2}$ minimum size in the left ventricle. Attenuation of LM, ascending aorta and left ventricle was measured on pre and post contrast images.

The noise was measured in the aorta $\left(100 \mathrm{~mm}^{2}\right.$ minimum size, located at the level of left main origin), air-filled cavities $\left(50 \mathrm{~mm}^{2}\right.$ in bronchia or trachea) and muscle $\left(25 \mathrm{~mm}^{2}\right.$, thoracic wall) in post-contrast images and was used to derive SNR and CNR values.

\section{Stenosis assessment and patient management}

The presence of significant stenosis ( $>50 \%$ of the lumen) in the 18 SCCT segments was reported by on-site radiologists using a 5 -point scale ( $5=$ certainly yes; $4=$ probably yes; $3=$ doubtful; 2 = probably no $; 1=$ certainly no).

On-site radiologists recorded the recommended management from the following list: no action, medication, invasive coronary angiography and other.

\section{Coronary track rate}

In order to determine whether image quality is sufficient for automated segmentation as often used for evaluation, commercially available software ("Comprehensive Cardiac Analysis" on IntelliSpace Portal, Philips) was used to automatically track LAD, LCX and RCA up to their distal segments and the number of segments tracked per patient constituted the 'coronary track 
rate', which was assessed by a third independent off-site radiologist, also with more than 10 years of experience in cardiac CT.

\section{Clinical safety}

All adverse events (AEs), including cardiac events as evidenced by ECG (performed up to 10 min post-injection), were reported from the patient's signature of the informed consent up to $30 \mathrm{~min}$ after the examination. The intensity of AEs was classified on a 3-point scale, based on interference with daily activities: mild (no interference), moderate (moderate interference) or severe (the subject is unable to work). The causal relationship of the AE to the CM injected was defined according to the French Method of Causality Assessment [30].

Patient comfort and pain were assessed on-site using a selfadministered questionnaire (5-point scale ranging from 1 (worst situation) to 5 (best situation)) and a visual analogue scale (VAS), respectively.

\section{Statistical analysis}

Assuming an expected proportion of $90 \%$ of patients with coronary CTAs evaluable for CAD diagnosis with the three investigated contrast agents, 424 assessable patients (3x141) were needed to ensure with $80 \%$ power and $5 \%$ two-sided type-one error that the lower limit of the $95 \%$ confidence interval $(95 \% \mathrm{CI})$ of the difference between iobitridol 350 and the best of the two comparators (iopromide 370 or iomeprol 400) is not greater than the clinical non-inferiority margin set at $-10 \%$.

Three patient populations were defined: all-includedpatients (AIP), full analysis set (FAS), and safety set.

AIP population included all patients enrolled in the study and having signed the informed consent. FAS included all patients who underwent the examination and had available assessments of the primary endpoint for iobitridol 350, iopromide 370 or iomeprol 400 examinations. The safety set included all patients who had received at least one injection of contrast agent, regardless of the quantity.

For comparisons between the three groups, two-sided tests were performed at a $5 \%$ level of significance. In case of multiple comparisons, significance level of each test was adjusted to ensure a $5 \%$ overall significance level. Accuracy of estimates was computed with $95 \%$ CIs.

Student's t-test and the F-test were used for quantitative variables, whereas the Chi-square test was used for qualitative variables.

Multiple regression models were performed to identify potential relationship between calcium scoring and stenosis assessment, image quality and territory.

\section{Results}

\section{Study population}

A total of 468 patients gave their consent and therefore were included (58\% male; aged $57.8 \pm 12.4$ years). Sixteen patients were excluded: five patients did not have a CM injection, and in 11 patients off-site image assessment was not possible due to technical failures. Therefore, 452 patients were analysed in the FAS and 463 in the safety set.

There were no significant differences between the three groups in terms of demographics, clinical symptoms, risk factors and pre-CTA heart rate. No differences were noted in terms of requirement for $\beta$ blockers for the CTA procedure, calcium score and radiation dose (Table 1).

\section{Image evaluation for detection of stenosis}

The rate of patients with evaluable CT scans was not significantly different between the three groups $(92.1 \%$, $95.4 \%$ and $94.6 \%$ of patients in the FAS, for iobitridol, iopromide and iomeprol, respectively) (Figure 1 and Table 2). The $95 \%$ CI of the difference between iobitridol and the best of the two comparators (iopromide) was [-8.8 to 2.1], demonstrating the non-inferiority of iobitridol, when compared to other $\mathrm{CMs}$, in its ability to allow CAD diagnosis through a complete assessment of coronary artery segments.

The average score for image quality per-segment (total number of segments $=6,220$ ) was $3.5 \pm 0.9,3.5 \pm 0.8$ and 3.4 \pm 0.9 for the iobitridol, iopromide and iomeprol groups, respectively $(\mathrm{p}>0.05)$.

\section{Attenuation, signal and noise measurements}

The average pre-contrast vascular attenuation calculated from values of the ascending aorta, LM and left ventricle was $42.2 \pm 9.7 \mathrm{HU}$, without any difference between the three groups $(p=0.993$; Table 3$)$. Vascular attenuation was significantly increased in post-contrast images as compared to pre-contrast images in all three structures. Average post-contrast arterial vascular attenuation was $426.3 \pm 92.9 \mathrm{HU}, 449.8 \pm 88.1 \mathrm{HU}$ and $466.4 \pm 104.6 \mathrm{HU}$ for the iobitridol, iopromide and iomeprol groups, respectively $(p=0.001)$. The difference between groups was statistically significant for absolute values; however, when values accounting for noise were plotted as SNR and CNR, differences were no longer significant (Table 3). Measurements of noise in the ascending aorta showed no significant difference between groups $(\mathrm{p}=0.311)$. 
Table 1 Patients and procedure characteristics

\begin{tabular}{|c|c|c|c|c|c|}
\hline Parameters & Iobitridol 350 & Iopromide 370 & Iomeprol 400 & Total & Test \\
\hline Demographics & $\mathrm{N}=155$ & $\mathrm{~N}=160$ & $\mathrm{~N}=153$ & $\mathrm{~N}=468$ & \\
\hline Age (years) & $57.9 \pm 12.2$ & $58.7 \pm 11.6$ & $56.9 \pm 13.4$ & $57.8 \pm 12.4$ & $\mathrm{p}=0.457(\mathrm{~F})$ \\
\hline Male gender & $90(58.1 \%)$ & $92(57.5 \%)$ & $88(57.5 \%)$ & $270(57.7 \%)$ & $\mathrm{p}=0.993(\mathrm{C})$ \\
\hline Height (cm) & $170.4 \pm 10.5$ & $168.9 \pm 8.9$ & $169.8 \pm 10.0$ & $169.7 \pm 9.8$ & $\mathrm{p}=0.380(\mathrm{~F})$ \\
\hline Body weight (kg) & $78.7 \pm 15.4$ & $76.6 \pm 16.9$ & $77.3 \pm 14.0$ & $77.5 \pm 15.5$ & $\mathrm{p}=0.445(\mathrm{~F})$ \\
\hline Body mass index $\left(\mathrm{kg} / \mathrm{m}^{2}\right)$ & $27.0 \pm 4.3$ & $26.7 \pm 4.7$ & $26.7 \pm 3.6$ & $26.8 \pm 4.2$ & $\mathrm{p}=0.736(\mathrm{~F})$ \\
\hline \multicolumn{6}{|l|}{$\mathrm{CV}$ risk factors } \\
\hline Hypertension & $79(51.0 \%)$ & $84(52.5 \%)$ & $82(53.6 \%)$ & $245(52.4 \%)$ & $\mathrm{p}=0.898(\mathrm{C})$ \\
\hline Diabetes & $15(9.7 \%)$ & $17(10.6 \%)$ & $17(11.1 \%)$ & $49(10.5 \%)$ & $\mathrm{p}=0.916(\mathrm{C})$ \\
\hline Smoking & $49(31.6 \%)$ & $63(39.4 \%)$ & $53(34.6 \%)$ & $165(35.3 \%)$ & $\mathrm{p}=0.347(\mathrm{C})$ \\
\hline Family history of CAD & $58(37.4 \%)$ & $62(38.8 \%)$ & $67(43.8 \%)$ & $187(40.0 \%)$ & $\mathrm{p}=0.484(\mathrm{C})$ \\
\hline Hyperlipidaemia & $65(41.9 \%)$ & $68(42.5 \%)$ & $64(41.8 \%)$ & $197(42.1 \%)$ & $\mathrm{p}=0.992(\mathrm{C})$ \\
\hline History of obesity (BMI $>30 \mathrm{~kg} / \mathrm{m}^{2}$ ) & $34(21.9 \%)$ & $32(20.0 \%)$ & $29(19.0 \%)$ & $95(20.3 \%)$ & $\mathrm{p}=0.804(\mathrm{C})$ \\
\hline \multicolumn{6}{|l|}{ Symptoms at inclusion } \\
\hline Typical angina & $41(26.5 \%)$ & $40(25.0 \%)$ & $36(23.5 \%)$ & $117(25.0 \%)$ & $\mathrm{p}=0.839(\mathrm{C})$ \\
\hline Atypical angina & $63(40.6 \%)$ & $73(45.6 \%)$ & $73(47.7 \%)$ & $209(44.7 \%)$ & $\mathrm{p}=0.439(\mathrm{C})$ \\
\hline Non-angina chest pain & $50(32.3 \%)$ & $47(29.4 \%)$ & $44(28.8 \%)$ & $141(30.1 \%)$ & $\mathrm{p}=0.774(\mathrm{C})$ \\
\hline Calcium score & $\mathrm{N}=151$ & $N=152$ & $\mathrm{~N}=149$ & $\mathrm{~N}=452$ & \\
\hline Total score (mean \pm SD; range) & $\begin{array}{c}154.0 \pm 353.4 \\
(0-2288)\end{array}$ & $\begin{array}{c}204.8 \pm 400.9 \\
(0-1972)\end{array}$ & $\begin{array}{c}135.6 \pm 330.5 \\
(0-1890)\end{array}$ & $\begin{array}{c}165.0 \pm 363.4 \\
(0-2288)\end{array}$ & $\mathrm{p}=0.231(\mathrm{~F})$ \\
\hline \multicolumn{6}{|l|}{ In classes } \\
\hline Normal (no calcium) & $65(43.0 \%)$ & $70(46.1 \%)$ & $72(48.3 \%)$ & $207(45.8 \%)$ & $\mathrm{p}=0.631(\mathrm{C})$ \\
\hline Mild (1-100) & $47(31.1 \%)$ & $34(22.4 \%)$ & $45(30.2 \%)$ & $126(27.9 \%)$ & \\
\hline Moderate (101-400) & $20(13.2 \%)$ & $25(16.4 \%)$ & $15(10.1 \%)$ & $60(13.3 \%)$ & \\
\hline High $(401-1,000)$ & $11(7.3 \%)$ & $13(8.6 \%)$ & $10(6.7 \%)$ & $34(7.5 \%)$ & \\
\hline Severe $(>1000)$ & $8(5.3 \%)$ & $10(6.6 \%)$ & $7(4.7 \%)$ & $25(5.5 \%)$ & \\
\hline Heart rate & $\mathrm{N}=147$ & $\mathrm{~N}=156$ & $\mathrm{~N}=152$ & $\mathrm{~N}=455$ & \\
\hline Before CTA (bpm), mean \pm SD (range) & $\begin{array}{r}61.6 \pm 9.0 \\
(45-113)\end{array}$ & $\begin{array}{c}61.2 \pm 9.1 \\
(39-92)\end{array}$ & $\begin{array}{c}62.0 \pm 9.4 \\
(37-90)\end{array}$ & $\begin{array}{l}61.6 \pm 9.2 \\
\quad(37-113)\end{array}$ & $\mathrm{p}=0.737(\mathrm{~F})$ \\
\hline During CTA (bpm), mean \pm SD (range) & $\begin{array}{l}61.5 \pm 9.7 \\
\quad(41-103)\end{array}$ & $\begin{array}{c}61.0 \pm 8.9 \\
(40-89)\end{array}$ & $\begin{array}{l}61.4 \pm 10.4 \\
(36-97)\end{array}$ & $\begin{array}{l}61.3 \pm 9.7 \\
\quad(36-103)\end{array}$ & $\mathrm{p}=0.893(\mathrm{~F})$ \\
\hline$\beta$-blockers & $\mathrm{N}=112$ & $\mathrm{~N}=111$ & $\mathrm{~N}=115$ & $\mathrm{~N}=338^{*}$ & \\
\hline Intravenous & $63(56.3 \%)$ & $60(54.1 \%)$ & $61(53.0 \%)$ & $184(54.4 \%)$ & $\mathrm{p}=0.885(\mathrm{C})$ \\
\hline Oral & $49(43.8 \%)$ & $51(45.9 \%)$ & $54(47.0 \%)$ & $154(45.6 \%)$ & \\
\hline Radiation dose (post-injection values) & $\mathrm{N}=151$ & $\mathrm{~N}=152$ & $\mathrm{~N}=149$ & $\mathrm{~N}=452$ & \\
\hline Dose-length product mean $\pm \mathrm{SD}$; (range) & $\begin{array}{l}415.3 \pm 312.3 \\
\quad(38.0-1360.0)\end{array}$ & $\begin{array}{l}445.9 \pm 323.0 \\
\quad(25.0-1562.0)\end{array}$ & $\begin{array}{l}458.8 \pm 307.1 \\
\quad(22.0-1437.0)\end{array}$ & $\begin{array}{l}439.9 \pm 314.1 \\
\quad(22.0-1562.0)\end{array}$ & $P=0.469(F)$ \\
\hline $\mathrm{CT}$ dose index mean $\pm \mathrm{SD}$; (range) & $\begin{array}{l}26.5 \pm 21.5 \\
\quad(0.0-101.0)\end{array}$ & $\begin{array}{l}28.4 \pm 20.7 \\
\quad(1.0-101.0)\end{array}$ & $\begin{array}{r}30.0 \pm 21.4 \\
(1.0-96.0)\end{array}$ & $\begin{array}{l}28.3 \pm 21.2 \\
\quad(0.0-101.0)\end{array}$ & $\mathrm{P}=0.373(\mathrm{~F})$ \\
\hline
\end{tabular}

Results are expressed as mean \pm standard deviation or $\mathrm{n}(\%)$ of patients

* Subgroup of patients who received $\beta$-blockers as premedication for CTA

$B M I$ body mass index, $C A D$ coronary artery disease, $C V$ cardiovascular, $C T$ computed tomography, $C T A$ CTangiography, $F$ F-test, $C$ Chi-square test

\section{Other secondary endpoints}

No difference was observed regarding the number of significant stenoses identified with the three CMs $(p=0.580$; Table 4).
Multivariate analyses showed a relationship between calcium scoring and stenosis assessment according to territory $(p<0.001)$ and between calcium scoring and image quality regardless of the territory $(p=0.007)$. 

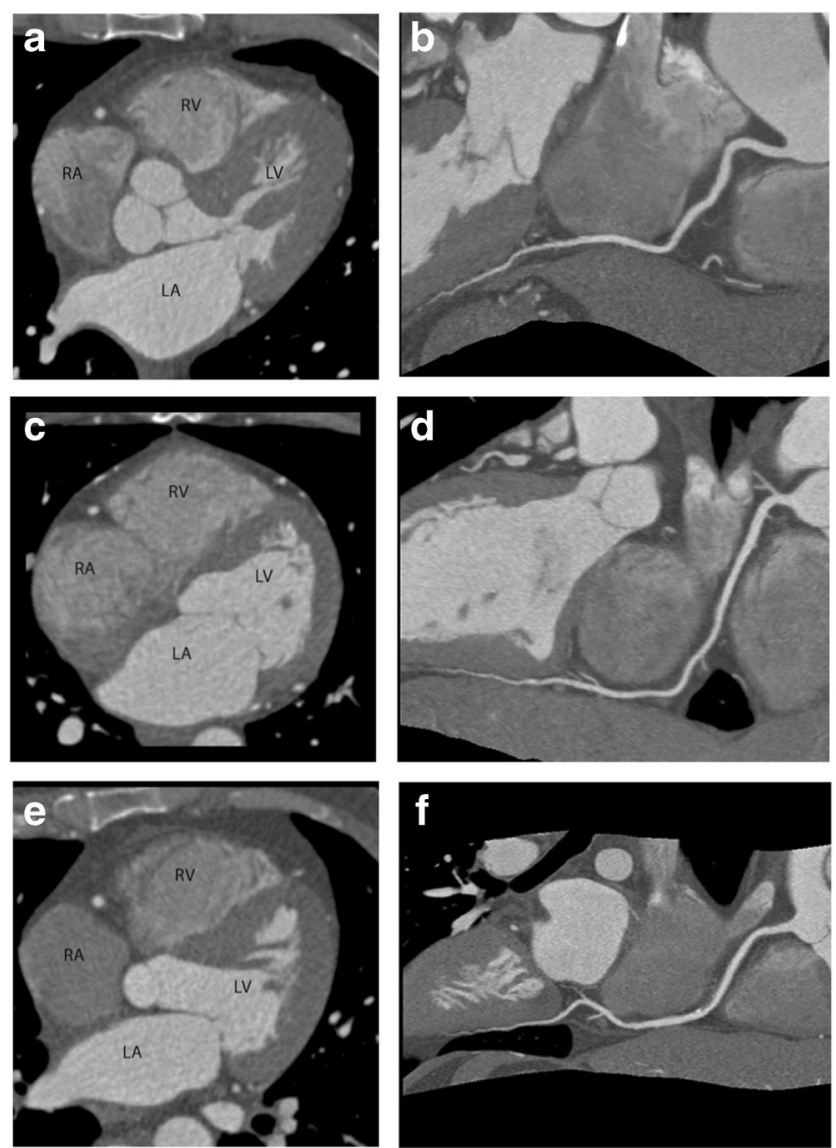

Fig. 1 Transaxial cross-sections (0.6-mm slice width) and curved multiplanar reconstructions of the right coronary artery, all displayed at a window level of 1,200 and width of $200 \mathrm{HU}$. (a, b) Investigation performed using iobitridol $350 \mathrm{mg} / \mathrm{ml}$. (c, d) Investigation performed using iopromide $370 \mathrm{mg} / \mathrm{ml}$. (e, f) Investigation performed using iomeprol $400 \mathrm{mg} / \mathrm{ml}$. $L A$ left atrium, $L V$ left ventricle, $R A$ right atrium, $R V=$ right ventricle

A mean of 11 segments were automatically tracked out of a maximum of 13 segments per patient whatever the contrast media injected (Table 4).

The mean score for comfort of the examination rated by the patient was good $(4.4 \pm 0.6)$ and similar for all three groups. Patient comfort was confirmed by a low reported intensity of pain (mean score of less than 1 out of $10 \mathrm{~cm}$ on VAS for the three groups).

Regarding patient management, no action was required after the CTA for $73 \%$ of the patients overall, with no significant difference between groups.

\section{Clinical safety}

The percentage of patients experiencing post CM-injection AEs was $15.1 \% ; 19.5 \%$ and $15.1 \%$, for the iobitridol, iopromide and iomeprol groups, respectively (Table 5). Most AEs concerned cardiac disorders, which were reported through systematic ECG follow-up performed up to $10 \mathrm{~min}$ post-injection. Overall, mean heart rate was $65.0 \pm 9.7 \mathrm{bpm} 2 \mathrm{~min}$ after $\mathrm{CM}$ injection and $62.8 \pm 9.3 \mathrm{bpm} 10 \mathrm{~min}$ after $\mathrm{CM}$ injection, and was similar in all three groups.

No severe AEs were reported. Only mild events were reported with iobitridol while four and seven moderate events were reported with iopromide and iomeprol, respectively. Few post-CM AEs were considered possibly related to CM administration: two in the iobitridol group and five in the iopromide group as well as in the iomeprol group. The cardiac events considered possibly related to CM injection were bradycardia (one patient in each group) and extrasystoles (two patients in the iomeprol group). Other possibly related events were pain in the iobitridol group, injection site pain, nausea, headache and urticaria in the iopromide group, injection site pain and feeling hot in the iomeprol group.

\section{Discussion}

The rationale for this trial was that $\mathrm{CM}$ iodine concentration may not play a role in the ability to visualize coronary stenoses by CTA. This study demonstrated the non-inferiority of iobitridol 350 in providing evaluable CT scans for assessment of coronary stenosis, as compared to CM with higher iodine concentrations (iopromide $370 \mathrm{mg}$ iodine/ml and iomeprol $400 \mathrm{mg}$ iodine/ml). These two CMs had been compared in a previous study without differences in terms of image quality [25]. Our study, the largest so far to address the relationship between iodine concentration and image quality, indicates the possibility of further reducing iodine content. Reflecting current clinical practice in many sites, IDR was not adjusted according to the iodine concentration of each CM.

Our results are in line with previous studies in which higher iodine concentrations were associated with higher intravascular attenuation [14, 31-34]. However, comparison is difficult since the volume of iodine injected was not always adjusted to patient weight [14]. The fact that no difference was observed between CMs when considering SNR and CNR cannot be fully explained. Compared to previous studies, image noise was relatively low given the advanced technology of the CT systems that were used. This limits the influence of noise. Also, statistically, variations in image noise across patients and systems may have been larger than variations in contrast enhancement, so that lack of significance regarding the difference between the three patient groups may be a statistical effect.

Recent improvements in terms of radiation dose management also affect vascular attenuation and noise. For instance, lower $\mathrm{kV}$ protocols $(80 \mathrm{kV})$ can now be used for patients with small body mass indices (BMIs) allowing for a reduction in radiation dose while increasing vascular attenuation [6]. In this study, no protocol using less than $100 \mathrm{kV}$ was performed. Another recent development affecting noise is related to the 
Table 2 Image quality per patient - off-site evaluation (FAS, $\mathrm{N}=452$ )

\begin{tabular}{|c|c|c|c|c|c|}
\hline & Iobitridol 350 & Iopromide 370 & Iomeprol 400 & All & Test \\
\hline Patient level & $\mathrm{N}=151$ & $\mathrm{~N}=152$ & $\mathrm{~N}=149$ & $\mathrm{~N}=452$ & \\
\hline Patients with evaluable CT scans & $139(92.1 \%)$ & $145(95.4 \%)$ & $141(94.6 \%)$ & $425(94.0 \%)$ & $\mathrm{p}=0.438(\mathrm{C})$ \\
\hline \multicolumn{6}{|l|}{ Number of non-diagnostic segments/patient } \\
\hline 0 & $139(92.1 \%)$ & $145(95.4 \%)$ & $141(94.6 \%)$ & $425(94.0 \%)$ & $\mathrm{p}=0.181(\mathrm{C})$ \\
\hline $1-5$ & $5(3.3 \%)$ & $3(2.0 \%)$ & $1(0.7 \%)$ & $9(2.0 \%)$ & \\
\hline $6-10$ & $4(2.6 \%)$ & $3(2.0 \%)$ & $4(2.7 \%)$ & $11(2.4 \%)$ & \\
\hline$>10$ & $3(2.0 \%)$ & $1(0.7 \%)$ & $3(2.0 \%)$ & $7(1.5 \%)$ & \\
\hline Average image quality mean $\pm \mathrm{SD}$; (range) & $3.5 \pm 0.9(0.0-4.0)$ & $3.5 \pm 0.8(0.2-4.0)$ & $3.4 \pm 0.9(0.0-4.0)$ & $3.5 \pm 0.9(0.0-4.0)$ & $\mathrm{p}=0.750(\mathrm{~F})$ \\
\hline Segment level & $\mathrm{N}=2082$ & $\mathrm{~N}=2090$ & $\mathrm{~N}=2048$ & $\mathrm{~N}=6220$ & \\
\hline \multicolumn{6}{|l|}{ Image quality } \\
\hline 0 - Non diagnostic & $89(4.3 \%)$ & $43(2.1 \%)$ & $71(3.5 \%)$ & $203(3.3 \%)$ & \\
\hline $1-$ Poor quality & $65(3.1 \%)$ & $82(3.9 \%)$ & $61(3.0 \%)$ & $208(3.3 \%)$ & \\
\hline 2 - Moderate quality & $143(6.9 \%)$ & $151(7.2 \%)$ & $187(9.1 \%)$ & $481(7.7 \%)$ & \\
\hline 3 - Good quality & $258(12.4 \%)$ & $313(15.0 \%)$ & $334(16.3 \%)$ & $905(14.5 \%)$ & \\
\hline 4 - Excellent quality & $1527(73.3 \%)$ & $1501(71.8 \%)$ & $1395(68.1 \%)$ & $4423(71.1 \%)$ & \\
\hline
\end{tabular}

$F$ F-test; $C$ Chi-square test

Table 3 Signal quantification at patient level - off-site evaluation (FAS, N =452)

\begin{tabular}{|c|c|c|c|c|c|}
\hline & $\begin{array}{l}\text { Iobitridol } 350 \\
\mathrm{~N}=151\end{array}$ & $\begin{array}{l}\text { Iopromide } 370 \\
\mathrm{~N}=152\end{array}$ & $\begin{array}{l}\text { Iomeprol } 400 \\
\mathrm{~N}=149\end{array}$ & $\begin{array}{l}\text { All } \\
N=452\end{array}$ & $\begin{array}{l}\text { p-value } \\
\text { (F-Test) }\end{array}$ \\
\hline \multicolumn{6}{|l|}{ Attenuation } \\
\hline \multicolumn{6}{|l|}{ Ascending aorta } \\
\hline Pre & $44.8 \pm 9.9$ & $44.2 \pm 10.3$ & $44.7 \pm 11.3$ & $44.6 \pm 10.5$ & 0.864 \\
\hline Post & $440.0 \pm 94.0$ & $465.0 \pm 93.6$ & $476.8 \pm 114.9$ & $460.6 \pm 102.2$ & 0.006 \\
\hline \multicolumn{6}{|l|}{ Left ventricle } \\
\hline Pre & $42.0 \pm 11.6$ & $41.1 \pm 10.7$ & $41.4 \pm 11.1$ & $41.5 \pm 11.1$ & 0.789 \\
\hline Post & $382.3 \pm 108.8$ & $401.2 \pm 113.7$ & $431.1 \pm 124.3$ & $404.7 \pm 117.2$ & 0.001 \\
\hline \multicolumn{6}{|c|}{ Left main coronary artery } \\
\hline Pre & $39.7 \pm 14.5$ & $41.1 \pm 15.7$ & $40.8 \pm 13.4$ & $40.5 \pm 14.6$ & 0.666 \\
\hline Post & $441.9 \pm 101.0$ & $466.8 \pm 100.8$ & $479.9 \pm 116.6$ & $462.8 \pm 107.2$ & 0.008 \\
\hline Average pre-contrast & $\begin{array}{l}42.2 \pm 9.7 \\
(21.1-90.5)\end{array}$ & $\begin{array}{c}42.2 \pm 10.2 \\
(15.3-92.0)\end{array}$ & $\begin{array}{l}42.3 \pm 9.4 \\
(19.9-84.6)\end{array}$ & $\begin{array}{l}42.2 \pm 9.8 \\
(15.3-92.0)\end{array}$ & 0.993 \\
\hline Average post-contrast & $\begin{array}{l}426.3 \pm 92.9 \\
\quad(240.6-726.2)\end{array}$ & $\begin{array}{l}449.8 \pm 88.1 \\
\quad(240.3-717.4)\end{array}$ & $\begin{array}{l}466.4 \pm 104.6 \\
\quad(185.5-766.9)\end{array}$ & $\begin{array}{l}447.4 \pm 96.6 \\
\quad(185.5-766.9)\end{array}$ & 0.001 \\
\hline Average noise aorta & $\begin{array}{l}32.5 \pm 10.5 \\
(15.5-80.5)\end{array}$ & $\begin{array}{c}32.8 \pm 10.0 \\
(13.8-69.2)\end{array}$ & $\begin{array}{l}34.3 \pm 11.5 \\
(14.3-75.7)\end{array}$ & $\begin{array}{l}33.2 \pm 10.7 \\
(13.8-80.5)\end{array}$ & 0.311 \\
\hline Average SNR & $\begin{array}{l}16.2 \pm 5.6 \\
{[7.0-45.7)}\end{array}$ & $\begin{array}{l}17.1 \pm 5.0 \\
(6.5-31.4)\end{array}$ & $\begin{array}{l}17.6 \pm 6.6 \\
(5.2-46.4)\end{array}$ & $\begin{array}{l}17.0 \pm 5.8 \\
(5.2-46.4)\end{array}$ & 0.109 \\
\hline Average CNR & $\begin{array}{l}14.4 \pm 5.4 \\
(5.6-42.8)\end{array}$ & $\begin{array}{l}15.3 \pm 4.8 \\
(5.8-28.7)\end{array}$ & $\begin{array}{l}15.8 \pm 6.5 \\
(4.3-48.0)\end{array}$ & $\begin{array}{l}15.2 \pm 5.6 \\
(4.3-48.0)\end{array}$ & 0.090 \\
\hline
\end{tabular}

All values are expressed as Hounsfield Units (HU) with mean and standard deviation. The range is provided in brackets for average values.

FAS full analysis set, CNR contrast to noise ratio, SNR signal to noise ratio, Pre prior to contrast media intravenous administration, Post after contrast media intravenous administration, $S D$ standard deviation 
Table 4 Secondary efficacy endpoints

\begin{tabular}{|c|c|c|c|c|c|}
\hline Secondary endpoints & Iobitridol 350 & Iopromide 370 & Iomeprol 400 & Total & Test \\
\hline Pain assessment & $\mathrm{N}=148$ & $\mathrm{~N}=148$ & $N=144$ & $\mathrm{~N}=440$ & \\
\hline $\begin{array}{l}\text { Pain during and after } \\
\text { examination - VAS }(\mathrm{cm})\end{array}$ & $0.4 \pm 0.9$ & $0.5 \pm 1.0$ & $0.7 \pm 1.5$ & $0.6 \pm 1.2$ & $\mathrm{p}=0.049(\mathrm{~F})$ \\
\hline Patient comfort & $\mathrm{N}=150$ & $\mathrm{~N}=151$ & $\mathrm{~N}=148$ & $\mathrm{~N}=449$ & \\
\hline Comfort score* & $4.4 \pm 0.6$ & $4.4 \pm 0.6$ & $4.3 \pm 0.6$ & $4.4 \pm 0.6$ & $\mathrm{p}=0.347(\mathrm{~F})$ \\
\hline Patient management & $\mathrm{N}=151$ & $\mathrm{~N}=152$ & $\mathrm{~N}=149$ & $\mathrm{~N}=452$ & \\
\hline No action & $107(70.9 \%)$ & $109(71.7 \%)$ & $114(76.5 \%)$ & $330(73.0 \%)$ & $\mathrm{p}=0.494(\mathrm{C})$ \\
\hline Medication & $22(14.6 \%)$ & $24(15.8 \%)$ & $19(12.8 \%)$ & $65(14.4 \%)$ & $\mathrm{p}=0.752(\mathrm{C})$ \\
\hline Invasive coronary angiography & $20(13.2 \%)$ & $18(11.8 \%)$ & $14(9.4 \%)$ & $52(11.5 \%)$ & $\mathrm{p}=0.572(\mathrm{C})$ \\
\hline Other recommendation & $6(4.0 \%)$ & $6(3.9 \%)$ & $8(5.4 \%)$ & $20(4.4 \%)$ & $\mathrm{p}=0.791(\mathrm{C})$ \\
\hline \multicolumn{6}{|l|}{ Stenosis $(>50 \%)$ assessment } \\
\hline Per-segment & $\mathrm{N}=2718$ & $N=2736$ & $\mathrm{~N}=2682$ & $\mathrm{~N}=8136$ & $\mathrm{p}=0.003(\mathrm{C})$ \\
\hline Certainly no & $1670(84.3 \%)$ & $1729(83.4 \%)$ & $1753(86.5 \%)$ & $5152(84.8 \%)$ & \\
\hline Probably no & $235(11.9 \%)$ & $263(12.7 \%)$ & $211(10.4 \%)$ & $709(11.7 \%)$ & \\
\hline Doubtful & $26(1.3 \%)$ & $32(1.5 \%)$ & $26(1.3 \%)$ & $84(1.4 \%)$ & \\
\hline Probably yes & $29(1.5 \%)$ & $17(0.8 \%)$ & $28(1.4 \%)$ & $74(1.2 \%)$ & \\
\hline Certainly yes & $20(1.0 \%)$ & $31(1.5 \%)$ & $8(0.4 \%)$ & $59(1.0 \%)$ & \\
\hline Per patient & $\mathrm{N}=151$ & $\mathrm{~N}=152$ & $\mathrm{~N}=149$ & $\mathrm{~N}=452$ & \\
\hline $\begin{array}{l}\text { overall significant stenosis } \\
\text { (doubtful to certainly) }\end{array}$ & $31(20.5 \%)$ & $30(19.7 \%)$ & $24(16.1 \%)$ & $85(18.8 \%)$ & $\mathrm{p}=0.580(\mathrm{C})$ \\
\hline Coronary track rate & $\mathrm{N}=151$ & $\mathrm{~N}=152$ & $\mathrm{~N}=149$ & $\mathrm{~N}=452$ & \\
\hline $\begin{array}{l}\text { number of tracked } \\
\text { segments per patient }\end{array}$ & $10.9 \pm 2.2$ & $10.8 \pm 2.4$ & $11.1 \pm 2.3$ & $10.9 \pm 2.3$ & \\
\hline
\end{tabular}

Results are expressed as mean \pm standard deviation or $\mathrm{n}(\%)$ of patients or segments

$V A S$ visual analogue scale $(10 \mathrm{~cm}), F$ F-test; $C$ Chi-square test

*Comfort during examination was rated by the patient on a scale from 1 (very poor) to 5 (very good)

Table 5 Incidence and characteristics of adverse events (safety set, $\mathrm{N}=463$ )

\begin{tabular}{lcccc}
\hline Adverse events & $\begin{array}{l}\text { Iobitridol } 350 \\
\mathrm{~N}=152\end{array}$ & $\begin{array}{l}\text { Iopromide } 370 \\
\mathrm{~N}=159\end{array}$ & $\begin{array}{l}\text { Iomeprol } 400 \\
\mathrm{~N}=152\end{array}$ & $\begin{array}{l}\text { All } \\
\mathrm{N}=463\end{array}$ \\
\hline $\mathrm{n}$ ( \%) of patients with at least one: & & & \\
$\quad$ Pre CM-injection AE & $24(15.8 \%)$ & $14(8.8 \%)$ & $15(9.9 \%)$ & $53(11.4 \%)$ \\
Post CM-injection AE & $23(15.1 \%)$ & $31(19.5 \%)$ & $23(15.1 \%)$ & $77(16.6 \%)$ \\
Number of post CM-injection AE & 26 & 36 & 28 & 90 \\
Intensity & & & & \\
$\quad$ Mild & $26(100.0 \%)$ & $32(88.9 \%)$ & $20(71.4 \%)$ & $78(86.7 \%)$ \\
$\quad$ Moderate & $0(0.0 \%)$ & $4(11.1 \%)$ & $7(25.0 \%)$ & $11(12.2 \%)$ \\
$\quad$ Severe & $0(0.0 \%)$ & $0(0.0 \%)$ & $1(3.6 \%)$ & $1(1.1 \%)$ \\
Outcome & & & & \\
$\quad$ Resolved & $26(100.0 \%)$ & $34(94.4 \%)$ & $27(96.4 \%)$ & $87(96.7 \%)$ \\
$\quad$ Ongoing & $0(0.0 \%)$ & $2(5.6 \%)$ & $1(3.6 \%)$ & $3(3.3 \%)$ \\
Relationship to CM & $12(46.2 \%)$ & $22(61.1 \%)$ & $18(64.3 \%)$ & $52(57.8 \%)$ \\
$\quad$ Not related & $12(46.2 \%)$ & $9(25.0 \%)$ & $5(17.9 \%)$ & $26(28.9 \%)$ \\
$\quad$ Doubtfully related & $2(7.7 \%)$ & $5(13.9 \%)$ & $5(17.9 \%)$ & $12(13.3 \%)$ \\
$\quad$ Possibly related &
\end{tabular}

$A E$ adverse events, $C M$ contrast media

One patient could have experienced several adverse events 
introduction of iterative reconstruction algorithms which are able to significantly reduce image noise thus improving SNR and CNR while keeping intravascular attenuation constant $[6$, 9]. It is therefore reasonable to expect that using lower $\mathrm{kV}$ settings and an iterative reconstruction algorithm may have yielded better SNR values regardless of other parameters. However, the inclusion of iterative reconstruction algorithms might have hampered comparisons to images from other scanners unless image noise would have been adapted by lowering the radiation exposure during the acquisition. Finally, newer detectors have been introduced with increased efficiency and lower noise [5] hence promoting the use of less iodine content (less volume or lower concentrations [35-40]).

Regarding safety, iobitridol use was associated with the lowest incidence of related AEs (i.e. $1.3 \%$ corresponding to two patients), confirming the excellent safety profile of this $\mathrm{CM}[41-43]$.

\section{Study limitations}

The main limitation of the study is the absence of a gold standard such as conventional angiography. Diagnostic accuracy could therefore not be compared between groups. A similar number of scans positive for stenosis in all three groups, however, indicate that there is likely no systematic difference in stenosis detection rates between the three CMs.

Another limitation is the relatively low coronary calcium burden. This is typical for the target population of the study. While severely calcified vessels may benefit from increased vessel lumen opacification for reliable evaluation $[16,17]$, this was not specifically evaluated and the ability to identify calcified lesions was not compared across the three CMs. A systematic assessment of renal function postcontrast was not performed. Further, contrast injection rates for the three CMs used were constant and not adjusted to achieve equal IDR across the three groups. Hence IDR ranged from 1,400 to $1,600 \mathrm{mg}$ iodine/s in the patients with the lowest body weight and from 2,100 to 2,400 mg iodine/ $\mathrm{s}$ in patients with the highest body weight. This reflects clinical practice, where protocols typically prescribe injection rates in $\mathrm{ml} / \mathrm{s}$ and not IDR, and explains why higher attenuation values were observed for higher concentration CMs, but did not lead to significant differences regarding SNR, CNR or the clinical rating of image quality.

\section{Conclusion}

With current CT technology, iobitridol $350 \mathrm{mg}$ iodine $/ \mathrm{ml}$ is not inferior to $\mathrm{CMs}$ with higher iodine in terms of image quality for coronary stenosis assessment by CTA. When considering image quality, SNR and CNR, iobitridol yielded similar values to iopromide and iomeprol. Iobitridol, with a lower content of iodine, holds the potential to reduce the risk of adverse reactions, as supported by its excellent safety profile. It is likely that developments in image reconstruction and detector technology may further allow improving image quality while minimizing the necessary amount of injected iodine.

Acknowledgments The scientific guarantor of this study is Stephan Achenbach. The authors of this manuscript declare relationships with the following companies:

Stephan Achenbach: Research Grants, Siemens Healthcare and Abbott Vascular. Consultant, Guerbet. Francois Laurent: Advisory Board, Guerbet.

Sebastian Leschka: Speaker Honoraria, Siemens Healthcare, GE Healthcare and Guerbet.

Didier Revel: Consultant, Guerbet and CSL Behrung. Salah Dine Qanadli: Consultant, Guerbet, Cordis, Cook and Bard. Filippo Cademartiri: Consultant, Guerbet, Bracco, Servier and Siemens Healthcare. Thel other authors have no conflicts of interest to disclose. This study received funding from Guerbet. No complex statistical methods were necessary for this paper.

Institutional Review Board approval was obtained. Written informed consent was obtained from all subjects (patients) in this study. Methodology: prospective, randomised controlled trial, multicentre study.

Open Access This article is distributed under the terms of the Creative Commons Attribution 4.0 International License (http:// creativecommons.org/licenses/by/4.0/), which permits unrestricted use, distribution, and reproduction in any medium, provided you give appropriate credit to the original author(s) and the source, provide a link to the Creative Commons license, and indicate if changes were made.

\section{References}

1. Rahsepar AA, Arbab-Zadeh A (2015) Cardiac CT vs. stress testing in patients with suspected coronary artery disease: review and expert recommendations. Curr Cardiovasc Imaging Rep 8

2. Roifman I, Rezai MR, Wijeysundera HC et al (2015) Utilization of cardiac computed tomography angiography and outpatient invasive coronary angiography in Ontario, Canada. J Cardiovasc Comput Tomogr 9:567-571

3. Al-Mallah MH, Aljizeeri A, Villines TC et al (2015) Cardiac computed tomography in current cardiology guidelines. J Cardiovase Comput Tomogr 9:514-523

4. Lell M, Marwan M, Schepis T et al (2009) Prospectively ECGtriggered high-pitch spiral acquisition for coronary CT angiography using dual source CT: technique and initial experience. Eur Radiol 19:2576-2583

5. Schuhbaeck A, Achenbach S, Layritz C et al (2013) Image quality of ultra-low radiation exposure coronary $\mathrm{CT}$ angiography with an effective dose $<0.1 \mathrm{mSv}$ using high-pitch spiral acquisition and raw data-based iterative reconstruction. Eur Radiol 23:597-606

6. Maffei E, Martini C, De Crescenzo S et al (2010) Low dose CT of the heart: a quantum leap into a new era of cardiovascular imaging. Radiol Med 115:1179-1207

7. Weustink AC, Neefjes LA, Kyrzopoulos S et al (2009) Impact of heart rate frequency and variability on radiation exposure, image quality, and diagnostic performance in dual-source spiral CT coronary angiography. Radiology 253:672-680

8. Weustink AC, Mollet NR, Neefjes LA et al (2009) Preserved diagnostic performance of dual-source CT coronary angiography with reduced radiation exposure and cancer risk. Radiology 252:53-60 
9. Gordic S, Desbiolles L, Sedlmair M et al (2016) Optimizing radiation dose by using advanced modelled iterative reconstruction in high-pitch coronary CT angiography. Eur Radiol 26:459-468

10. Dey D, Schuhbaeck A, Min JK, Berman DS, Achenbach S (2013) Non-invasive measurement of coronary plaque from coronary CT angiography and its clinical implications. Expert Rev Cardiovasc Ther 11:1067-1077

11. Fischer C, Hulten E, Belur P, Smith R, Voros S, Villines TC (2013) Coronary $\mathrm{CT}$ angiography versus intravascular ultrasound for estimation of coronary stenosis and atherosclerotic plaque burden: a meta-analysis. J Cardiovasc Comput Tomogr 7:256-266

12. Cademartiri F, La Grutta L, Palumbo AA et al (2006) Coronary plaque imaging with multislice computed tomography: technique and clinical applications. Eur Radiol 16:M44-M53

13. Agatston AS, Janowitz WR, Hildner FJ, Zusmer NR, Viamonte M Jr, Detrano R (1990) Quantification of coronary artery calcium using ultrafast computed tomography. J Am Coll Cardiol 15:827-832

14. Becker CR, Vanzulli A, Fink C et al (2011) Multicenter comparison of high concentration contrast agent iomeprol-400 with iso-osmolar iodixanol-320: contrast enhancement and heart rate variation in coronary dual-source computed tomographic angiography. Investig Radiol 46:457-464

15. Cademartiri F, Mollet NR, Lemos PA et al (2006) Higher intracoronary attenuation improves diagnostic accuracy in MDCT coronary angiography. AJR Am J Roentgenol 187:W430-W433

16. Cademartiri F, Maffei E, Palumbo AA et al (2008) Influence of intra-coronary enhancement on diagnostic accuracy with 64-slice CT coronary angiography. Eur Radiol 18:576-583

17. Cademartiri F, Runza G, Mollet NR et al (2005) Impact of intravascular enhancement, heart rate, and calcium score on diagnostic accuracy in multislice computed tomography coronary angiography. Radiol Med 110:42-51

18. Fleischmann D (2005) How to design contrast injection protocols for multiple detector-row CT angiography (MDCTA). Eur Radiol 15:E60-E65

19. Cademartiri F, Mollet NR, van der Lugt A et al (2005) Intravenous contrast material administration at helical 16-detector row CT coronary angiography: effect of iodine concentration on vascular attenuation. Radiology 236:661-665

20. Katzberg RW, Barrett BJ (2007) Risk of iodinated contrast material-induced nephropathy with intravenous administration. Radiology 243:622-628

21. Thomsen HS, Morcos SK, Barrett BJ (2008) Contrast-induced nephropathy: the wheel has turned 360 degrees. Acta Radiol 49:646-657

22. Nyman U, Almen T, Aspelin P, Hellstrom M, Kristiansson M, Sterner G (2005) Contrast-medium-Induced nephropathy correlated to the ratio between dose in gram iodine and estimated GFR in $\mathrm{ml} / \mathrm{min}$. Acta Radiol 46:830-842

23. Cademartiri F, de Monye C, Pugliese F et al (2006) High iodine concentration contrast material for noninvasive multislice computed tomography coronary angiography: iopromide 370 versus iomeprol 400. Investig Radiol 41:349-353

24. Rist C, Nikolaou K, Kirchin MA et al (2006) Contrast bolus optimization for cardiac 16-slice computed tomography: comparison of contrast medium formulations containing 300 and 400 milligrams of iodine per milliliter. Investig Radiol 41:460-467

25. Kim EY, Yeh DW, Choe YH, Lee WJ, Lim HK (2010) Image quality and attenuation values of multidetector CT coronary angiography using high iodine-concentration contrast material: a comparison of the use of iopromide 370 and iomeprol 400 . Acta Radiol 51:982-989

26. Cademartiri F, Nieman K, van der Lugt A et al (2004) Intravenous contrast material administration at 16-detector row helical CT coronary angiography: test bolus versus bolus-tracking technique. Radiology 233:817-823

27. Cademartiri F, van der Lugt A, Luccichenti G, Pavone P, Krestin GP (2002) Parameters affecting bolus geometry in CTA: a review. J Comput Assist Tomogr 26:598-607

28. Rumberger JA, Sheedy PF 2nd, Breen JF, Fitzpatrick LA, Schwartz RS (1996) Electron beam computed tomography and coronary artery disease: scanning for coronary artery calcification. Mayo Clin Proc 71:369-377

29. Raff GL, Abidov A, Achenbach S et al (2009) SCCT guidelines for the interpretation and reporting of coronary computed tomographic angiography. J Cardiovasc Comput Tomogr 3:122-136

30. Begaud B, Evreux JC, Jouglard J, Lagier G (1985) Imputation of the unexpected or toxic effects of drugs. Actualization of the method used in France. Therapie 40:111-118

31. Bae KT, Heiken JP, Brink JA (1998) Aortic and hepatic contrast medium enhancement at CT. Part II Effect of reduced cardiac output in a porcine model. Radiology 207:657-662

32. Bae KT, Heiken JP, Brink JA (1998) Aortic and hepatic contrast medium enhancement at CT. Part I. Prediction with a computer model. Radiology 207:647-655

33. Bae KT, Heiken JP, Brink JA (1998) Aortic and hepatic peak enhancement at CT: effect of contrast medium injection ratepharmacokinetic analysis and experimental porcine model. Radiology 206:455-464

34. Bae KT, Tran HQ, Heiken JP (2000) Multiphasic injection method for uniform prolonged vascular enhancement at CT angiography: pharmacokinetic analysis and experimental porcine model. Radiology 216:872-880

35. Lembcke A, Schwenke C, Hein PA et al (2014) High-pitch dualsource CT coronary angiography with low volumes of contrast medium. Eur Radiol 24:120-127

36. Lell MM, Jost G, Korporaal JG et al (2015) Optimizing contrast media injection protocols in state-of-the art computed tomographic angiography. Investig Radiol 50:161-167

37. Oda S, Utsunomiya D, Yuki $\mathrm{H}$ et al (2015) Low contrast and radiation dose coronary CT angiography using a 320-row system and a refined contrast injection and timing method. J Cardiovasc Comput Tomogr 9:19-27

38. Yin WH, Lu B, Gao JB et al (2015) Effect of reduced x-ray tube voltage, low iodine concentration contrast medium, and sinogramaffirmed iterative reconstruction on image quality and radiation dose at coronary CT angiography: results of the prospective multicenter REALISE trial. J Cardiovasc Comput Tomogr 9:215-222

39. Mangold S, Wichmann JL, Schoepf UJ, et al (2016) Automated tube voltage selection for radiation dose and contrast medium reduction at coronary CT angiography using 3rd generation dualsource CT. Eur Radiol. Feb 4

40. Kim R, Park EA, Lee W, Chung JW (2016) Feasibility of 320-row area detector $\mathrm{CT}$ coronary angiography using $40 \mathrm{~mL}$ of contrast material: assessment of image quality and diagnostic accuracy. Eur Radiol. Feb 23

41. Petersein J, Peters CR, Wolf M, Hamm B (2003) Results of the safety and efficacy of iobitridol in more than 61,000 patients. Eur Radiol 13:2006-2011

42. Vogl TJ, Honold E, Wolf M, Mohajeri H, Hammerstingl R (2006) Safety of iobitridol in the general population and at-risk patients. Eur Radiol 16:1288-1297

43. Wendt-Nordahl G, Rotert H, Trojan L et al (2006) Intravenous contrast media in uroradiology: evaluation of safety and tolerability in almost 50,000 patients. Med Princ Pract 15:358-361 